\title{
Role of Nanoparticles in Latent Fingerprinting: An Update
}

\author{
Kajol Bhati 1,*(D), Divya Bajpai Tripathy ${ }^{1(\mathbb{D})}$ \\ 1 Division of Forensic Science, SBAS, Galgotias University, Greater Noida, Uttar Pradesh-201312, India; \\ divyabaj@gmail.com (D.B.T); \\ * Correspondence: bhati.kajol18@gmail.com;
}

Received: 15.06.2020; Revised: 5.07.2020; Accepted: 6.07.2020; Published: 8.07.2020

\begin{abstract}
Nanotechnology is an escalating branch whose feelers are mesmerizing nearly every field of science. It is the evolving field in forensic science, the advancement of emergent techniques of nanoparticles being unified with latent fingerprinting. The size of nanomaterials made them unique and have adjustable mechanical, electrical, and optical properties. At the crime scene, the prints that are left behind are generally latent (invisible) fingerprints. The Current review paper encompasses the synthesis of nanoparticles from conventional and advances synthetic pathways. Their applications, with and without modifications and their impacts on the enhancement of latent fingerprints, have also been discussed.
\end{abstract}

Keywords: Nanoparticles; latent prints; fingerprints; nanopowder; crime scene; evidence.

(C) 2020 by the authors. This article is an open-access article distributed under the terms and conditions of the Creative Commons Attribution (CC BY) license (https://creativecommons.org/licenses/by/4.0/).

\section{Introduction}

Nanotechnology is one of the advanced branches of science that is focused on nuclear, sub-atomic, and supramolecular particles planning to make nanostructures with improved functionalities [1]. The term nanoparticle depicts particulate issues running in size from 1-100 $\mathrm{nm}$ [2]. Nanotechnology is an escalating branch whose feelers are mesmerizing nearly every field of science. With the improvement of nanotechnology, it has been found nanomaterials are generally utilized in criminological sciences for investigation and testing. Nanotechnology is the evolving field in forensic science, the advancement of emergent techniques of nanoparticles being unified with latent fingerprinting. The size of nanomaterials made them unique and have adjustable mechanical, electrical, and optical properties [3]. Their size correspondingly permits them to have a brilliant and large reactivity and offers the capability to be modified by an innumerable of functional agents. The nanostructures aids in the detection and expansion of latent fingerprints. Different nanoparticles such as $\mathrm{Si}, \mathrm{C}, \mathrm{Al}, \mathrm{Cu}, \mathrm{Ba}, \mathrm{Fe}, \mathrm{V}, \mathrm{Au}$, and $\mathrm{Ag}$ have been industrialized to obtain latent fingerprints. The general and individual characteristics of fingerprints and other minute details like the position of pores are enhanced by nanoparticles.

In general, at the crime scene, the most common but invisible evidence left behind is the fingerprints of the suspect as well as of the victim. These fingerprints give almost exact verification of the presence of a person at the crime sight, as every human being has a unique pattern of a fingerprint. These fingerprints can be easily distinguished based on their dissimilar ridges and patterns. Fingerprints obtained due to the sweat secreted by the glands named as eccrine and apocrine existing on the fingers. Although these glands are present throughout the body and responsible for the secretion of sweat, help in leaving the traces of invisible prints at 
the scene of crime due to the presence of ridges and whorls present on fingerprint during the process of deposition [4]. Fingermarks, consisting of water, amino acids, fatty acids, and minerals, could be defined as imprints of regions left on a surface of friction ridge skin through different analytical methods [5].

Powder dusting method is the earliest technique used to develop invisible fingerprints $[6,7]$, which is still a preferable one at the crime scene as it is cost-effective, easily available, and ease in use. However, the traditional powder-dusting method has some disadvantages in terms of low sensitivity, less contrast, toxic in nature, and high autofluorescence intrusion. Therefore, to overcome these disadvantages and to get more accurate and precise results to use of nanoparticles has come into practice as a better choice. In the same direction, various researchers and scientists contributed by developing nanoparticles, studying their exploitation in the enhancement of invisible fingermarks with better character development.

\section{Methods}

Since now, many different types of nanoparticles have been synthesized and exploited in latent fingerprinting such as Aptamer- functionalized core-shell nanoparticle, Iron Oxide Nanoparticles, etc. They have been found to have different results in fingerprints applications, as discussed below:

\subsection{Aptamer-functionalized core-shell nanoparticle.}

The Aptamer is an oligonucleotide that is short in length and single-stranded in nature. Owing to have such characteristics, this oligosaccharide can be mold into a 3-D structure in the existence of target to apprehend precise recognition these unsurpassed properties, like easy modification in structure, high affinity, and specificity, flexible design, biochemical stability, slightly synthetic make Aptamer, a very good choice to be used in latent fingerprint development. These carbohydrate molecules easily get bonded with lysozymes, a universal compound present in human sweat [8].

A lysozyme binding aptamer modified structure by fusing $\mathrm{Au} / \mathrm{PNTP} / \mathrm{SiO}_{2}$ and studied them using surface-enhanced Raman scattering (SERS). The study revealed that specific molecules (PNTP) implanted amongst the gold core and the shell of silica were effective in evading the interference of the external environment. This helped in determining the reproducibility and stability of SERS indicators. In accumulation to that, the free surface of the silica shell could be modified with Lysozyme Binding Aptamer (LBA).

Zhao et al., 2016 [9] used Fren's method to prepare Au nanoparticles [10], Synthesized $\mathrm{Au}$ nanoparticles, and $\mathrm{Au} / \mathrm{PNTP} / \mathrm{SiO}_{2}$ lysozyme binding aptamer nanoparticles have been characterized using the transmission electron microscope, Raman spectroscopy spectra were measured using UV- Visible spectrophotometer. Modified sandwiched SERS probes have been used to effectively detect the developed and recorded latent fingerprints from the glass surface, results obtained revealed that these nanoprobes might easily be placed on the ridges of fingermark and recognized at all the three levels, pattern, individual characteristics, and pores in fingerprints were visible using these nanoparticles [11].

Fingerprints are a shred of important evidence. At a crime scene, usually latent prints are present. To develop these prints, the most common method is the powder- dusting technique, a traditional method [12]. It has low sensitivity, background interference, low contrast. The need for alternative methods leads to the development of many other alternative 
techniques. Nanoparticles played an important role as one of the alternative techniques. Fluorescent nanoparticles (NPs) like upconversion nanoparticles (UCNPs) and quantum dots (QDs) have a great property which helps in developing latent fingerprints with better sensitivity and contract since it has some exceptional properties like large surface area, high fluorescent intensity, small particle size, and photochemical stability [13, 14, 15, 16, 17].
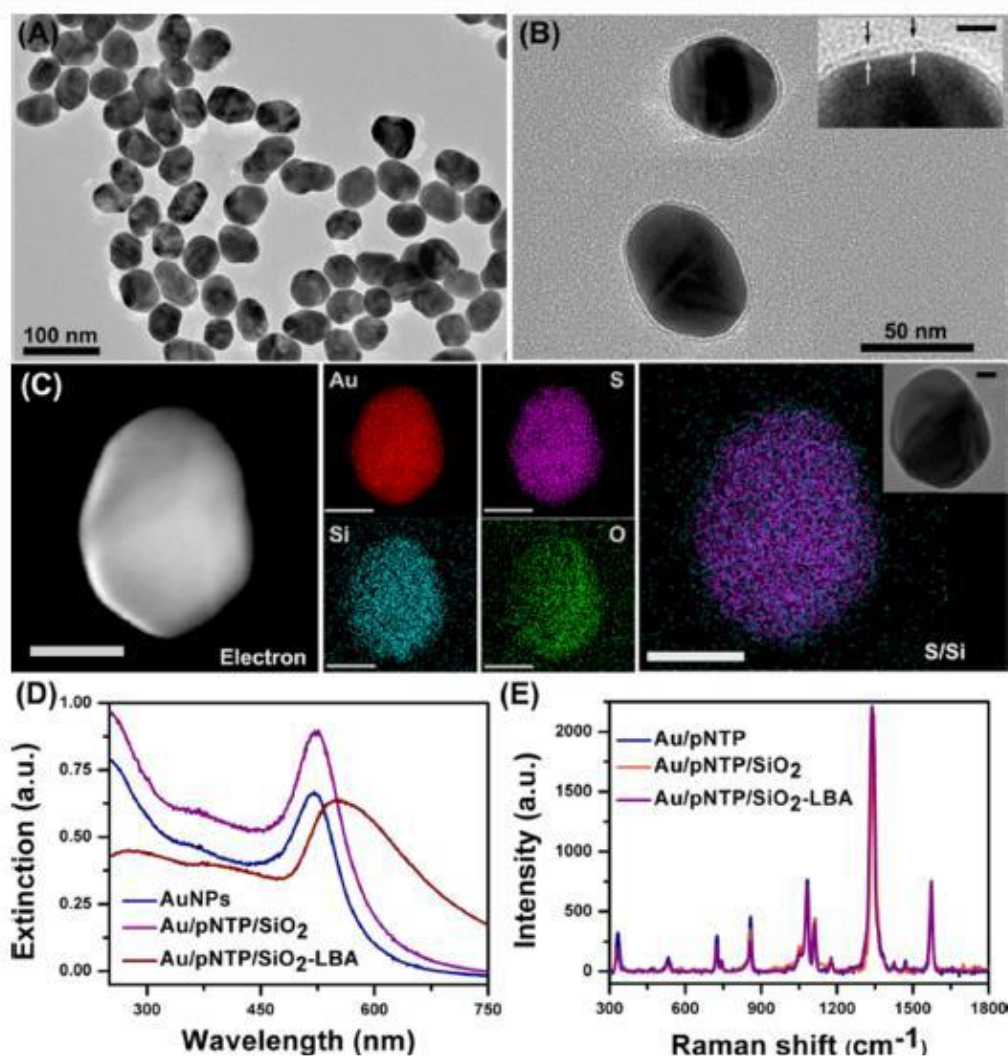

Figure 1. The Transmission electron microscopy pictures of a) Nanoparticles of gold b) Gold /pNTP/Silicon Dioxide SERS nanoprobes c) STEM images of NPs (Au/pNTP/SiO2) using electron, Gold, Sulphur, Silica, and Oxygen signals and the STEM photographs (superimposed image) of Sulphur and Silica. (D) Extinction spectrum of $\mathrm{Au} / \mathrm{pNTP} / \mathrm{SiO}_{2} \mathrm{SERS}$ nanoprobes (purple line), pure Au NPs (navy line), and $\mathrm{Au} / \mathrm{pNTP} / \mathrm{SiO}_{2}$-LBA SERS nanoprobes (red line). (E) SERS spectrum of Au/pNTP/SiO 2 SERS nanoprobes (orange line), pNTP-functionalized AuNPs (blue line) and Au/pNTP/SiO ${ }_{2}-\mathrm{LBA}$ SERS nanoprobes (violet line) [9]. Reprinted with permission from https://doi.org/10.1021/acsami.6b03352. Copyright (2016) American Chemical Society.

Using of UCNPs showed a better result than any other nanoparticles, they can convert the long wavelength radiation into shorter wavelength emission by the mechanism of multiphoton $[17,18,19,20,21]$. These nanoparticles get excited by near-infrared light to release strong visible fluorescence. Hence, with the help of UCNPs, sensitivity, and contrastenhanced, and also remove the interference of the background while detecting the latent fingerprints.

On the contrary, quantum dots uses ultraviolet radiation, which results in the fluorescence of substrate and substantial autofluorescence interference [22, 16, 23 24, 25]. Whereas in UCNPs the use of Near-Infrared radiation to discharge visible light avoids the autofluorescence interfering by the substratum. Fingerprints do contain DNA, which is safe under NIR radiation and is not as much injurious to the eyes and skin of the handler than the UV light. $\mathrm{NaYF}_{4}: \mathrm{Yb}, \mathrm{Er}^{39}$, and $\mathrm{YVO}_{4}: \mathrm{Yb}, \mathrm{Er}^{40}$ upconversion luminous particles can be used on invisible fingermarks for its development, on numerous semi-permeable and impermeable 
surfaces and the solution is commercially available for use. Due to the non- uniform size and shape, less dispersion and size fluctuating between $\sim 0.2$ to $2 \mu \mathrm{m}$ leads to the reduction of affinity between the fingerprint's residues and particles and decreased detection sensitivity. A research done by Wang et al. on nano particles of $\mathrm{NaYF}_{4}$ : $\mathrm{Yb}, \mathrm{Er}$ to checked the working of the particles with lysozyme-binding Aptamer for lysozyme recognition in the fingerprint residues for latent fingerprints detection. This technique confined complex steps and was impractical to use at the scene of crime since it needed the modification in the chemical of upconversion nanoparticles $(\sim 260 \mathrm{~nm})$ by complicated measures. The gestation of finger impressions was for 30 mins in UCNPs solution.

Another method developed by Wang et al. designed for the fusion of $\mathrm{NaYF}_{4}: \mathrm{Yb}, \mathrm{Er}$ fluorescent upconversion nanoparticles (UCNPs) using the oleic acid-based solvothermal method with the maximum conceivable fluorescence strength beneath near-infrared (NIR) treatment [26]. The factors like volume, time, optimization, and reaction temperature of oleic acid have been studied for the composition phase, and size, and intensity of the UC fluorescence of the upconversion nanoparticles [20]. The subsequent UCNPs have been used to fluorescently label the fingerprints as there might be a chance of UCNPs release the visible light if the excitation of near-infrared light reached to $980 \mathrm{~nm}$. The Dormant fingerprints were examined on significant sorts of even substrates, incorporating with a solitary foundation shading (white clay tiles, black marbles, and transparent glass), various foundation hues (marbles contained composite surface designs), and solid foundation autofluorescence (note, Chinese money, and plates made up of plastic). These results were equated with the traditional powder dusting methods that resulted that UCNPs showed better sensitivity, good contrast quality, less autofluorescence interference, and low background inference. Hence, this research showed that upconversion nanoparticles incorporated beneath upgraded conditions are an adaptable fluorescent marker for the effortless advancement of finger impressions and can locate their handy applications in criminological science[27].

\subsection{Iron oxide nanoparticles.}

Iron oxide nanoparticles stick to the ridges of fingerprints that help in the visualization of invisible finger impressions [28]. It has the capability of size modification to a different color varies as for un-annealing it showed black, and for annealing, it is brown. The powder form of nanoparticles has been used, which helped in the development and recognition of invisible finger impressions due to the physical affinity with fingermark deposits like sebum, sweat, and other contaminants. Annealed and unannealed iron oxide nanoparticles were synthesized by a green pathway, characterization has been done by XRD, and the purity of nanoparticles was confirmed by EDAX measurement [29]. Latent finger impressions were developed on permeable and impermeable surfaces (glass) [30]. The result showed betterdeveloped fingerprints on the absorbent surface as compared to the non-absorbent surfaces [31 32].

\subsection{Carbon dot-based nanopowders.}

A highly rapidly developing class of nanoparticles made from carbon $(\mathrm{C} \mathrm{dot})$, behaved as high-performance nano emitters showed strength for bioimaging, multicolor printing, catalyst, and act as sensors. C- dots are synthesized by a green pathway, which helped in making it cost-effective. They're biocompatible in nature and non-toxic to the environment and 
humans. C- dots improve the colloidal stability and enhance the fluorescence intensity. Because of the tendency of the pure sort of c- dots to aggregate in powder form and according to the reports, the $\mathrm{c}$ dots powder isn't photoactive because of wide-ranging clustering and metallurgical properties. Thanks to this tendency of c-dot, a hybrid nanopowder is ready to possess an occasional concentration of it and a serious portion of titania, silica, laponite, and white fingerprint powder. This helped within the flowability of powder and unique photoluminescent ofc- dots. The ridge patterns are visible using the hybrid nanopowder. The fingerprints developed shows some tuneability in color as in reflex to the incident beam wavelength. This showed the blue, green, or red appearance of the fingerprints once excited within the violet, blue, and green wavelength regions, respectively [5].

Similarly, the patterns of color were attained, consuming $0.7 \mathrm{wt} \% \mathrm{c}$-dots laponite of fingerprints left on metal and glass surfaces. In contrast, the detection of any kind of signals was not observed when c-dots was absent in another experiment. Using C-dot nanopowder, fingerprints were developed on beverage bottle foil that showed deprived contrast underneath high field radiance, and only faded patterns of ridges were visible underneath the green light. At an equivalent time, the better-developed features were determined under violet and blue radiation [33].

\section{4. $\mathrm{Cu}^{2+}$ engrafted $\mathrm{MgAl}_{2} \mathrm{O}_{4}$ nanoparticles.}

The synthesize of $\mathrm{Cu}^{2+}$ engrafted $\mathrm{MgAl}_{2} \mathrm{O}_{4}$ Nanoparticles have been done by the combustion method using chelating ligand Triethanolamine. The characterization was done by TEM and XRD methods, and the optical property has been checked by UV-DRS method. Magnesium showed photocatalytic activity, which helps in enhancing the latent fingerprints under UV light by brush method. The latent fingerprints were developed and enhanced by applying the powder onto the even surfaces like metallic and lead crystal and later on visualized under UV light at $254 \mathrm{~nm}$. The quality of enhanced prints was not good; the individual characteristics and pores were not visible [34].

\subsection{Calcium Molybdate nanophosphors.}

Metal Molybdates has a property of transferring the energy from the host lattice to the small ions, which makes it the one kind of oxide-based substance that owns the brilliant luminescent possessions. The metal molybdate having a space group of 141/a with transparent quality and permits different light range to undergo without showing any changes in the luminescence effect is Calcium Molybdate. This material owns adequate chemical and physical features as related to the other oxide materials. The $\mathrm{CaMoO}_{4}$ synthesis process takes a time of $12 \mathrm{~h}$, with the particle size ranges between 1-2 micron. This work developed, a comparatively speed up the production of nanostructures shaped as spherical molded with building blocks of nanoparticles [35]. The optical, structural characteristics have been considered of the Nanopowders, which proficiently detect the invisible finger impression on various surfaces [36]. The portrayal has been done by XRD, SEM, EDX. FTIR. The $\mathrm{CaMoO}_{4}$ was incapacitated with $\mathrm{Eu}^{3+}$ and $\mathrm{Tb}^{3+}$ ions, and for the recognition of invisible fingerprints, the properties of luminescence have been observed in it. Ridge details in the fingerprints were efficiently detected by it. The latent prints could be observed under visible light with better contrast and less interference of background. Different non-porous surfaces were taken to develop latent fingerprints, i.e., compact disc, glass slide, and stainless-steel cup. Therefore, using phosphor 
materials, the invisible finger impressions can be developed and detected on various surfaces. The fingerprints were developed by applying the nanopowder on the surface using a brush. The nanopowder was glued to the ridges of fingerprints, not to the grooves. Hence, it showed the minute ridges and sweat pores as well with good contrast and less interference of background. The presence of oily components and moisture in the latent fingerprints enhanced the affinity of the powder towards it. The calcium molybdate reacts with the minute quantity of amino acids existing in the invisible finger marks with a better affinity toward it. Hence, even after keeping the finger marks in the ambient atmosphere for 9 days, the prints were visible [36].

\section{6. $\mathrm{SiO}_{2} @ \mathrm{LaOF}: \mathrm{Eu}^{3+}$ core-shell functional nanomaterials.}

Silica is a material that is used in core-shell designed constituents and can adjust the size on its own. The nanoparticles are acted as shells that are coated with the silica leads to the formation of phosphors core-shell that is spherical in shape. With few changes or fabrication in the conditions of the experiment, a non-agglomerated, sphere-shaped element can be prepared. The prepared nanoparticles help to get the enhanced brightness with good resolution and reduction of light scattering. The particular nanoparticles can be used for different applications like anti-counterfeiting, biomedical imaging, and other kinds of display devices. The core-shell nanoparticles as these are rare-earth doped nano phosphors showed some specific features like shrill absorption and line emission in an ultra-violet visible spectrophotometer with a good yield of quantum, having exceptional biocompatibility, no toxicity, stability as a thermal and chemical product is good, elongated life and advanced photostability. The lanthanide ions with trivalent luminescence can simply be unified into the crystal assembly as matrices with fluoride as a host, and even at room temperature, it shows luminescence with high efficiency.

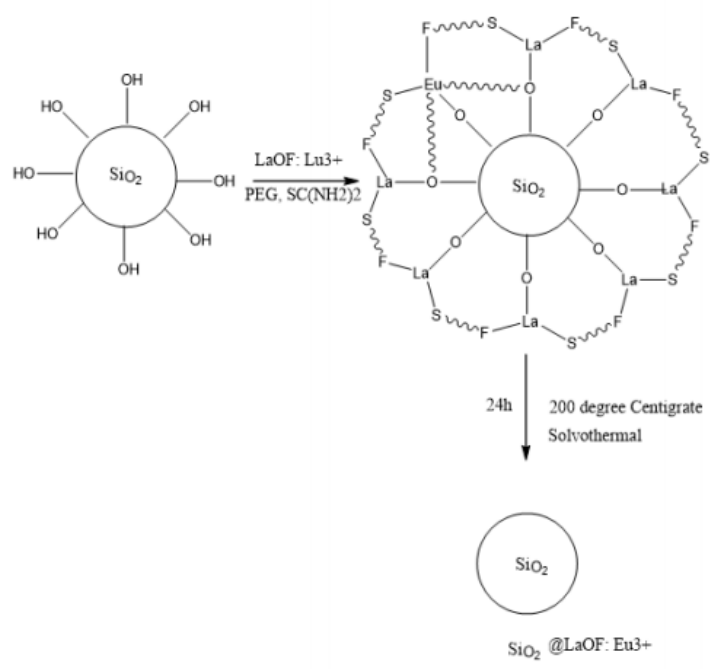

Figure 2. Production of $\mathrm{SiO}_{2} @ \mathrm{LaOF} \mathrm{Eu}^{3+}$ shell nanostructure.

$\mathrm{Eu}^{3+}$ ions can act as the finest dopant used in numerous hosts for the production of red color discharge, considered as long photoluminescence. The refractive index is higher in these particular nanomaterials. To change or reduce the distance between luminescent and quenching centers of nanomaterials, decrease in the defects of surfaces and reduction of non-radiative channels, surface modification method is one of the utmost accomplished technique to be used. These changes lead to the improvement in luminescent quality in a core-shell nanostructure. Rare-earth doped nanomaterials with not as much of than $100 \mathrm{~nm}$ got more consideration as 
surface-based research specifically in invisible finger impressions acknowledgment. The characterization has been done by XRD, SEM, TEM, EPR.

Using $\mathrm{SiO}_{2} @ \mathrm{LaOF}: \mathrm{Eu}^{3+}$ nanomaterials, the latent fingerprints were developed on the impermeable surface like scale made up of metal, tin foil, and a stapling device. It was detected the level 2 ridge structures were not visible under white light, but those ridge structures were visible underneath $254 \mathrm{~nm}$ Ultra violet rays. The nanopowder was castoff on several absorbent materials like deck cards, covers of a journal notes with background interference observed at $254 \mathrm{~nm}$ under UV light, and the ridge characteristics were visible. For the confirmation of fluorescent powders efficiency, different covered composite invisible finger impressions were taken on tin foil surface and stained with $\mathrm{SiO}_{2} @ \mathrm{LaOF}$ : $\mathrm{Eu}^{3+}$ nanoparticles and observed using white light and $254 \mathrm{~nm}$ ultra-violet light. All the three levels were visible under both the lights that showed the efficiency of fluorescent tagging agent for the imagining of fingerprints. The invisible finger impressions stained on tin foil kept from day 1 to 1 month. After a week, the prints were not evidently visible on the surface [37].

$\mathrm{SiO}_{2}$ nanoparticles were synthesized for the development of invisible finger impressions on non-absorbent surfaces. The method that was used for the synthesize was reversed microemulsion, where water droplets filled with dye played the role of the microreactor, permitting the growth of nanoparticles [38]. Triton X-100 (TX-100), ammonium hydroxide, Tetraethyl orthosilicate (TEOS), tris(2,20- bipyridyl) dichlororuthenium (II) hexahydrate (RuBpy) cyclohexane, n-hexanol, rhodamine 6G, Carbox- yethylsilanetriol sodium salt (CES) rhodamine B (Dye), sodium chloride $(\mathrm{NaCl})$, and 3-(triethoxysilyl)propylsuccinic anhydride (TES-PSA) has been used for the synthesizing process. This method has an advantage as it helps in controlling the acute size of the particle, acquaint with dye in the matrix and functionalizing the surface of nanoparticles. After that, nanoparticles were out of the emulsion, and further washed with ethanol and dissolved in water [38]. The characterization was done by dynamic light scattering, laser doppler micro-electrophoresis, and fluorescence spectrophotometer. The fingerprints were taken by three donors (two males and one female) on four different non-porous surfaces (glass, black polyethylene, aluminum foils, and transparent polypropylene) 20 samples were taken on each surface by one donor. The prints were developed using $\mathrm{SiO}_{2}$ nanoparticles that have luminescent properties due to the 3 dyes. The results of the developed prints were compared with the one-step luminescent cyanoacrylate process LumicyanoTM. The fingerprints have been cut into two parts, one part was treated using $\mathrm{SiO}_{2}$ nanoparticles, and another part was using LumicyanoTM. A correlation with LumicyanoTM, an advanced cyanoacrylate with luminescence, was then led in the direction of a survey to check the productivity of the technique. The two methods were thought about on the arrangement of twenty depletive imprints by 3 benefactors on 4 distinctive impermeable products. All things considered, the two systems performed likewise. Be that as it may, for LumicyanoTM, quality varieties between contributors were watched. Strikingly, that was not watched (or just to a constrained reach out) for $\mathrm{SiO}_{2}$ nanoparticles. This may be because of the way that the system focuses on a particular practical gathering rather than specific mixes of the emission. The $\mathrm{SiO}_{2}$ results quality was rather increasingly observed with the category of product. Diverse identification results demonstrated that the strategy stayed to be additionally advanced. The concerning and security problem regarding $\mathrm{SiO}_{2}$ nanoparticles showed that there was not one lethality evaluation of the blended nanoparticles was acted right now. This ought to be considered in subtleties before any enormous scope application. More work despite everything should be embraced to give a completely operational procedure, yet the revealed 
outcomes right now that $\mathrm{SiO}_{2}$ nanoparticles are extremely encouraging and that examination exertion ought to be additionally sought after [13].

\subsection{Aluminum oxide nanoparticles $\left(\mathrm{Al}_{2} \mathrm{O}_{3}-\mathrm{NPS}\right)$.}

The nanoparticles of Aluminum oxide were synthesized using a green pathway with a coating of eosin yellow (dye) and extract of seed. The method was non-toxic and eco-friendly, which helped in the invisible finger impression development. Cyamopsis tetragonoloba seed extract worked as a hydrophobic compound that resisted particles of water and aided the powder particles to stick onto the greasy substance of the invisible finger impressions. This helped in giving better visibility of prints on numerous non-porous and porous surfaces. This also helped in the identification of faded latent fingerprints [32].

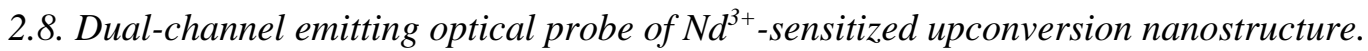

The nano phosphors synthesized using Lanthanide upconversion are beneficial to use in various fields, as it helps in reducing the fluorescence interference in the background and high signal to noise ratio of upconversion luminescence. The $\mathrm{Nd}^{3+}$ sensitized $\mathrm{NaYbF}_{4}$ :

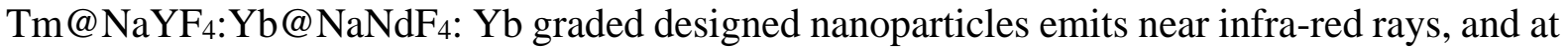
696 and $980 \mathrm{~nm}$, its luminescence and excitation occurred at $808 \mathrm{~nm}$ [39]. The $\mathrm{NaYbF}_{4}$ core endorses effective zeal transmitted towards $\mathrm{Tm}^{3+}$. The interlayer of $\mathrm{NaYF}_{4}$ : Yb effectively averts the process of cross-relaxation from $\mathrm{Tm}^{3+}$ to $\mathrm{Nd}^{3+}$. Therefore, it helped in improving the production of luminescent property. The $\mathrm{Nd}^{3+}$ helped in the alteration of wavelength excitation from 980 to $808 \mathrm{~nm}$.

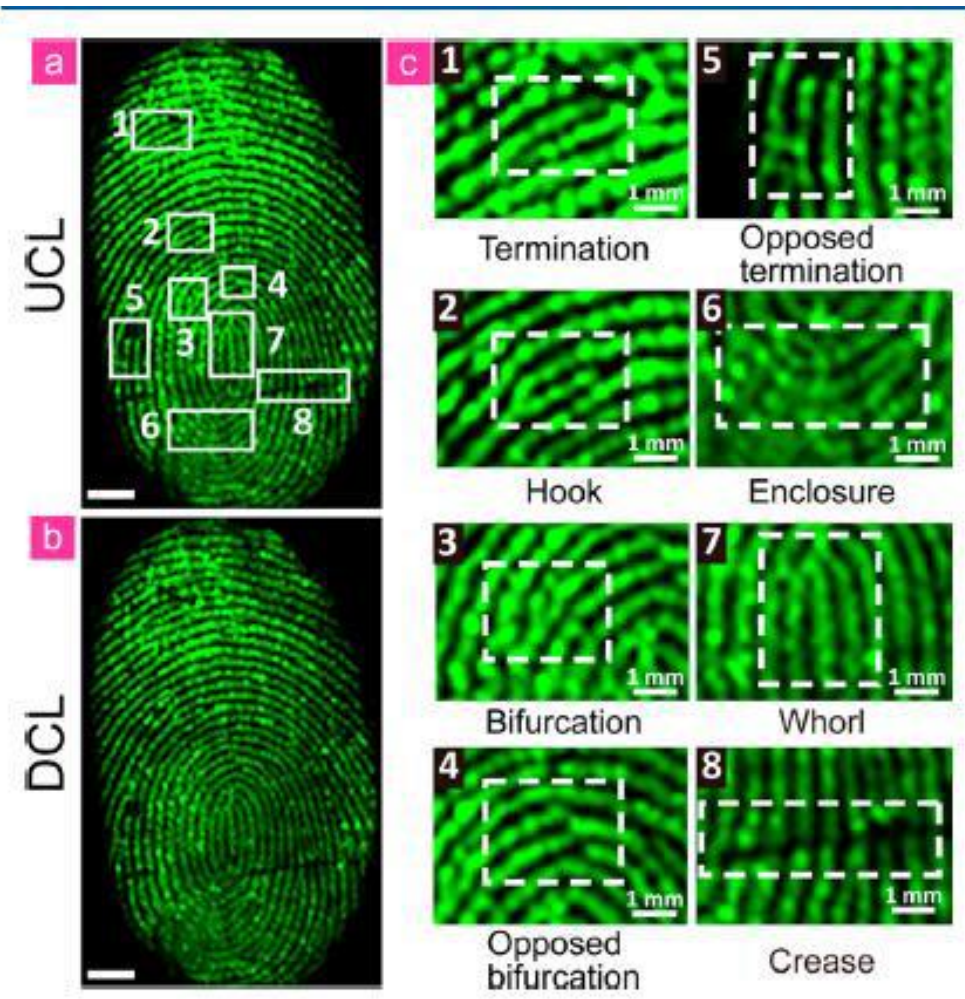

Figure 3. Stokes and UCL discharge pictures $(a, b)$ of finger impression on the surface of polyethylene baggage. (C)distended UCL images of fingerprints showed (1) termination, (2) hook, (3) bifurcation, (4) divergent bifurcation, (5) divergent termination (6) enclosure, (7) whorl, (8), and crease at $808 \mathrm{~nm}$ [39]. Reprinted with permission from https://doi.org/10.1021/acs.inorgchem.6b01536. Copyright (2016) American Chemical Society. 
The thermal effect has been faintly deterred induced by laser and leads to a new way for the secretion way of near infra-red rays at $980 \mathrm{~nm}$. The developed nanoparticles were used for invisible fingerprints and for bloody fingerprints image development, which showed details distinctly beneath $808 \mathrm{~nm}$ excitation and exhibited a huge signal to noise ratio with zero thermal damage to the fingerprint sample. These kinds of nanoparticles can be used in forensic applications for better enhancement of prints [39].

\subsection{Use of $N$-doped carbon dots for the imaging of finger impressions.}

Polyvinylpyrrolidone (PVP) polymers have been used for the $\mathrm{N}$ doped carbon dots synthesis, using the hydrothermal method, which helps in yielding the beaming fluorescent waves when excited at $350 \mathrm{~nm}$. These carbon dots were used for the recognition of finger impressions, and it showed the inimitable characteristics depicted as a clear, bright figure of the fingerprint. Chemical examination of $\mathrm{N}$-doped carbon dots showed that the surface functional group is $\mathrm{CeNHe}$, which contributes as a reason for the biomolecule's interaction with them, which led to the formation of precise fluorescence designs. The fluorescence emanation, initiated from the various fluorophores' designs, depicted three important components present on the $\mathrm{N}$ - carbon dots nanoparticles surface. They are non-hazardous in nature if the quantity is about $0.01 \mathrm{mg} / \mathrm{mL}$. In this particular work, the carbon dots were used on two substances as fluorescence tags to image finger impressions: tweezers and on the plastic surface. The result showed satisfactory finger impression images, showing clear ridges in the emission of orange color obtained with the help of filters helped in transmitting the lengthy wavelength to reduce the background contained fluorescence and allowed detailed imaging when illuminated at $360 \mathrm{~nm}$. The subsequent copy must be snapped with the help of a filter that illuminates the similar wavelengths as the one castoff for observing [40], for easy comparison and recognition of finger impression minutiae [41].

\subsection{Silver nanoparticles.}

A practical and easy technique was developed by Qin in 2013 [42] for visualization of latent fingerprints processed by eccrine and sebaceous glands by using silver and gold nanoparticles produced by the electrochemical process. The electrodeposition approach [43, 44] has been used as it only occurs on the valleys present between the ridges of fingerprints and the free conductive surface. This process enhanced the contrast and showed greater advantage as it is a simple, high resolution, non-hazardous, and rapid procedure used in the enhancement of invisible fingerprints on rough, smooth, clean, dirty conductive exteriors performed in aqueous solution hence, appropriate on a damp piece of evidence [45]. Different metal surfaces like stainless steel, platinum were taken, and silver and gold were electrodeposited on the metallic substrate between the elevations of fingerprints. The result showed clear images of invisible finger impressions on clean metal substrates in comparison to the dirty surface.

Due to the affinity of metallic silver towards the organic elements in fingerprint residue, it has been used in the physical developer method as a reagent since 1970. This helps in invisible fingermarks visualization on absorbent shells. The silver physical developer method is based on the oxidation-reduction reaction, where iron salt reduces the silver nitrate to metallic silver in an aqueous solution. The formation of silver nanoparticles takes place during the interaction of organic elements of fingerprint residue leads to the visualization of 
fingermark as a black silver image or dark grey on the surface. The development occurs due to the attraction of electrophoresis between the silver colloids (negatively charged) and fingerprint residues (positively charged). For porous surfaces, the silver physical developer method is appropriate as it visualized the prints on the moistened samples. The visibility of prints is not satisfactory. Therefore, before the dealing of prints with a silver physical developer, it has been treated with nanoparticles of gold using citrate ions as a stabilizer [32].

\subsection{Zinc Oxide Nanoparticles (ZnO-NPs).}

The properties of zinc oxide show high excitation binding energy $(60 \mathrm{MeV})$, and wide bandgap $(3.37 \mathrm{eV})$, this helps in the transition of zinc oxide at room temperature and contains an epoxy resin asset which enables the collaboration between the proteins and lipids present as residues of fingerprints on room temperature. Zinc oxide was used in the form of Nanopowders to enhance the old hidden finer impressions on impermeable exteriors. It helped in developing unique ridge details of fingerprints that fluoresced under UV light. According to the research done by Sydney University, Zinc oxide nanopowders develop clear prints as well as fluoresce naturally under UV light in wet conditions. Other studies showed that it also gives better fluorescence image when illuminated under long-wave UV light [32].

Arshad A et al. [46] synthesized $\mathrm{ZnO}-\mathrm{SiO}_{2}$ Nanopowder by the conventional heating method using $\mathrm{Zn}\left(\mathrm{CH}_{3} \mathrm{COO}\right)_{2} .2 \mathrm{H}_{2} \mathrm{O}, \mathrm{Na}_{2} \mathrm{SiO}_{3} .5 \mathrm{H}_{2} \mathrm{O}$, and $\mathrm{NaOH}$. The characterization has been done by FTIR, XRD, energy dispersive X-ray (EDX) analysis, Scanning electron microscope, and TEM. The size of the particles of $\mathrm{ZnO}-\mathrm{SiO}_{2}$ nanopowder done by TEM was $32.9 \mathrm{~nm}$. The enhancement of dormant finger impressions was done on dissimilar semi-permeable and nonporous substrates such as calculator, board marker, laptop with the help of small particle reagent, and powder dusting methods. SPR method has also been used on damp impermeable substrates. They exhibited clear quality prints with $2^{\text {nd }}$ and $3^{\text {rd }}$ level minutiae of ridges when compared to the commercial powders they gave better visibility and just stick to the ridges, unlike white powder which adhered to all over the surface.

Luthra $\mathrm{D}$ et al. helped in developing the latent fingerprints by synthesizing new metal oxides nanoparticles (tin oxide and zinc oxide) using chemical precipitation method in the form of dry powder for zinc oxide nanoparticles synthesis zinc acetate and $\mathrm{NaOH}$ were taken, and for tin oxide nanoparticles, tin chloride, and $\mathrm{NaOH}$ has been used followed by ethanol. The characterization has been done by X-ray diffraction, UV-Visible spectroscopy, and FTIR. The $\mathrm{ZnO}$ crystals size remained $14.75 \mathrm{~nm}$, while tin oxide was found to be $90 \mathrm{~nm}$ in size. These powders were used on the impermeable substrates like lead crystal, plastic, glossy cardboard, where dormant finger impressions existed, and they developed them. The study showed that Zinc oxide nanoparticles gave clear and better-enhanced fingerprints on all the three surfaces as compared to the prints developed by tin oxide nanoparticles [47].

The development of a latent finger impression on plastic surfaces has been successively done by the Vacuum Metal Deposition method using Au and Zn particles. A streamlined vacuum deposition process was directed to create a unique mark in this investigation. Unadulterated Zinc oxide has thermally vanished in a void framework, Zinc oxide could gather on polyethylene terephthalate (PET) surface. While zinc has been used to create a unique dormant mark in Gold/Zinc vacuum metal deposition, the subsequent Zinc store mostly reacted to Zinc oxide $(\mathrm{x})$ while coming in contact with air or during the deposition of zinc within sight of $\mathrm{O}_{2}$ [48]. In the interim, consistent Zinc oxide films kept by vacuum dissipation procedures were legitimately covered onto non-metallic substances on different things [49, 50, 51]. 
Subsequently, it is beneficial to utilize the vacuum statement of $\mathrm{ZnO}$ to create a unique mark on plastic surfaces. In this examination, the warm vanishing of $\mathrm{ZnO}$ was led to create a unique idle mark on polyethylene terephthalate, deprived of utilizing gold groups as the seed layer. Unique mark improvement on polyethylene terephthalate by Zinc oxide affidavit and Gold/Zinc vacuum metal deposition was thought about. No advancement of a unique mark is seen over polyethylene terephthalate after unadulterated $\mathrm{Zn}$ statement, deprived of using gold kernels. The outcome concurred with writing [52], demonstrating a poor testimony capacity of $\mathrm{Zn}$ on the plastic surface. The unique finger impression created by the gold/zinc vacuum metal deposition procedure utilizing gold and zinc products displays a solid difference between unique finger impression edges and valleys. The gold/zinc vacuum metal deposition procedure showed a typical improvement of a unique mark in metallic sparkle. Typical improvement with a clear differentiation between unique finger impression edges and valleys is too created by zinc oxide testimony. Dark unique mark is created using zinc oxide affidavit. It showed that Zinc oxide might be straightforwardly kept on the valley amongst unique finger impression edges and the free surface of polyethylene terephthalate. Along these lines, other than solitary metallic vacuum metal deposition [53] using vacuum vanishing of Zinc oxide can likewise create inert finger impressions on the polyethylene terephthalate substrates, lacking earlier smearing a slight coating of gold, and without pre-treatment by cyanoacrylate raging. The zinc oxide developed a clear pattern on fresh samples as compared to old samples. However, the zinc oxide deposition can create a unique preferable mark over gold/zinc vacuum metal deposition on the finger impression, matured for 1 month and 15 days [54].

\subsection{Gold nanoparticles (AuNPs).}

In the advancement of the dormant finger impressions process, Gold nanoparticles (AuNPs) played a noteworthy job due to their dormant behavior, better discernment, affectability, and qualities, that empower the capacity of the created finger impressions for a drawn-out timeframe [55]. In the multi-metal deposition, the gold nanoparticles were used due to their significant properties, which improve the permeability of the Latent fingerprints by two-advance sol-gel technique. The substrate that has a unique finger impression was absorbed using the gold nanoparticles solution (balanced out in the medium of citrate ion) trailed by the expansion of silver physical developer (Ag-PD solution) [15, 55]. Using a UV-Vis spectrophotometer, characterization has been done. The nanoparticles of gold bound to the residues of finger impressions and mobilize the precipitation of $\mathrm{Ag}$ particles to metallic $\mathrm{Ag}$ [46, 14, 56]. Due to the electrostatic interaction between the cationic charged finger impression residue and anionic charged Au nanoparticles, a silver picture of the dormant unique finger impression is acquired. Though, the use of the multi-metal deposition technique is constrained as it needs the item having the fingerprints to be washed in a fluid arrangement of the nanoparticles of gold. Consequently, the technique isn't appropriate for building up the prints on surfaces, for example, dividers and floors, or for any item too enormous to ever be absorbed a work area shower. This technique is expensive [32].

Nanomaterials have been used to develop latent fingerprints as it shows better enhancement of latent prints. It increases the superficial interface with the endogenic material present on the elevations, also enhances the disparity and finger impressions. As most of the scientists focused on visualized the fingerprints at a physical level, this paper focused on the exploration of molecular-level information embedded in latent fingerprints. A key trademark is the predominant presentation of nanomaterials in upgrading the affectability as well as 
particularity in estimation sciences [57, 58]. When gold metal is nanosized, it shows unmistakable visual characters because of the surface plasmon resonance (SPR) [59]. By altering the size, the shape of gold nanoparticles, a range of blue to red color can be obtained. The interesting property was utilized for detection as a colorimetric, surface-upgraded Raman scattering, and bioimaging. The light of gold nanoparticles with ultraviolet light can instigate quick warming in a little amount of the nanoparticles. The particular character can encourage the proficient desorption/ionization of particles accumulate on the surface of gold nanoparticles. The Nanoparticles of gold have dual properties which have been integrated. The characterization of AuNPs was done by TEM, and imaging of dormant finger impressions was done using the sputtering of gold. The coordination of certain characteristics of nanoparticles made up of gold was performed by imaging mass spectrometry for the depiction and atomic imaging of dormant finger impressions. Two differentiating hues (blue and pink), emerging from diverse surface plasmon resonance (SPR) groups of the gold nanoparticles, uncover the visual pictures of dormant finger impressions. The laser desorption/ionization characters of the gold nanoparticles permit the immediate investigation of endogenic and exogenic mixes inserted in dormant finger impressions and imaging their appropriations lacking upsetting the unique mark designs. The concurrent perception of dormant finger impressions and the account for the atomic pictures give proof on a singular way of life as well as settle covering fingerprints and recognize risky substances. The technique depicted the turn of events of a visual picture of dormant finger impressions in the parched condition. It can save compound data implanted in the unique mark for successive investigation by mass spectrometry. The various types of gold nanoparticles showing diverse surface plasmon resonance characters permit a distinct representation of dormant finger impressions by examination with unaided eyes. The laser desorption/ ionization property of gold nanoparticles makes it a successful mechanism for the straight examination of little particles inside invisible fingermarks and the age of atomic pictures. The reciprocal idea of the twofold pictures of invisible fingermarks, that is, ocular and atomic pictures, gives the physical example of fingerprints for singular ID and uncovers substance data inside fingerprints for scientific examinations. The gold nanoparticles electrical conductivity recently revealed by examining under the scanning electron microscope (SEM) for the examination of fingerprints at minuscule scale, that the AuNPs could go about as a viable mechanism for crossing over various imaging methods from plainly visible to infinitesimal (Scanning electron microscopy assessment) stages and uniform to atomic examination (mass spectrometric imaging) [60].

\subsection{Oligomer/silica hybrid nanoparticles.}

The red-emissive silica nanoparticles were synthesized by doping it with conjugated Oligomer Fluorescence Dioxaborolane Benzothiadiazole (OFDBT), which displayed an auspicious act on imaging of invisible finger impressions. The epoxy groups played a significant part in the preparation procedure because of its morphological and optical properties, as compared to the hydroxyl or amino groups modification. Three types of nanoparticles were developed using epoxy, amino, and a hydroxyl group. The Oligomer Fluorescence Dioxaborolane Benzothiadiazole /Silica Oxide-Epoxy nanoparticles were organized by the reverse micelle method using APTMS as one of the compounds, and the ultimate product was obtained by freeze-drying in the form of pink color powder. The Oligomer Fluorescence Dioxaborolane Benzothiadiazole /Silicon dioxide-OH nanoparticles were also prepared by reverse micelle method using silane as a component, collected by freeze-drying in 
the form of red powder. The synthesis of Oligomer Fluorescence Dioxaborolane Benzothiadiazole /Silicon Dioxide- $\mathrm{NH}_{2}$ nanoparticles was also alike to the process of Oligomer Fluorescence Dioxaborolane Benzothiadiazole/Silicon Dioxide-Epoxy nanoparticles but using 3-aminopropyl trimethoxy silane instead of 3-glycidyloxypropyl trimethoxy silane, and the concluding product was obtained by freeze-drying as pink powder. The characterization of nanoparticles has been done by DLS, Scanning electron microscope, NMR, MALDI, ultraviolet visible spectrophotometer. The Oligomer Fluorescence Dioxaborolane Benzothiadiazole /Silicon Dioxide-Epoxy nanoparticles displayed a sphere-shaped of $20 \mathrm{~nm}$ in size, useful to intermingle with finger impressions elevations through the mechanical procedure. The quantum yield and photostability of Oligomer Fluorescence Dioxaborolane Benzothiadiazole were amended expressively, due to the property of molecularly dispersing property of silica nanoparticles. The attained Oligomer Fluorescence Dioxaborolane Benzothiadiazole /Silicon Dioxide-Epoxy nanopowder exhibited kinship towards the emissions of the fingerprint's material, formed the pictures with fluorescence characters with enhanced firmness and features with detailing in it.

The stability of the nanoparticles with better sensitiveness showed a great visualization of dormant fingermarks in an image. The Oligomer Fluorescence Dioxaborolane Benzothiadiazole $/ \mathrm{SiO}_{2}-\mathrm{OH}$ NPs showed sphere-shaped particles of $42 \mathrm{~nm}$ size, but there was no clear accumulation because of the feeble collaboration of nanoparticles and insolvent such as water. The asymmetrical shapes of these nanoparticles were noticed, which occurred due to the excess number of amino groups. Similarly, the size distribution was detected in water using dynamic light scattering. The size of Oligomer Fluorescence Dioxaborolane Benzothiadiazole /Silicon Dioxide-Epoxy nanoparticles was around $25 \mathrm{~nm}$. In contrast, the other set of nanoparticles exhibited a wide range in size with a peak of almost 280nm. The hydrodynamic sizes of Oligomer Fluorescence Dioxaborolane Benzothiadiazole /Silicon Dioxide-OH nanoparticles and Oligomer Fluorescence Dioxaborolane Benzothiadiazole Silica nanoparticles were measured to be about 50 and $120 \mathrm{~nm}$, respectively. They showed comparable outcomes with scanning electron microscopy result outcomes. The light microscopy and fluorescence microscopy outcomes also established the propensity of accumulation below the parched condition of OFDBT/Silicon Dioxide-Epoxy nanoparticles. In contrast, Oligomer Fluorescence Dioxaborolane Benzothiadiazole /Silicon Dioxide-OH nanoparticles were additionally single spread, and the bulky amount of powder was detected in Oligomer Fluorescence Dioxaborolane Benzothiadiazole / Silicon Dioxide- $\mathrm{NH}_{2}$ nanoparticles.

To affirm the association type, the invisible finger impressions kept on lead crystal was submerged in $\mathrm{H}_{2} \mathrm{O}$ for twenty minutes and sixty minutes, at that point, created by the 3 sorts of nanoparticles. Nearly hydrophilic substrates may be liquified inside the drenching in $\mathrm{H}_{2} \mathrm{O}$, and further expelled as submersion time expanded. The relating imaging outcomes illustrated that every last one of the ocular and fluorescent fingermarks pictures created by the 3 nanoparticles indicated all around settled fingermarks over the lead crystal. At the point when the finger impressions were inundated in $\mathrm{H}_{2} \mathrm{O}$ for twenty minutes, and sixty minutes, the fluorescent pictures created by Oligomer Fluorescence Dioxaborolane Benzothiadiazole /Silicon DioxideEpoxy nanoparticles just demonstrated a small decrease inside the fluorescent force, and distinct finger impressions with better contrast were detected. A considerable modification was seen as the aftereffects of Oligomer Fluorescence Dioxaborolane Benzothiadiazole /Silicon Dioxide-OH nanoparticles. An unmistakable decrease in fluorescence signal was observed 
after twenty minutes inundation, and a blurred unique finger impression was detected after sixty minutes of submersion, which was hard to see by unaided eyes. The outside of OFDBT/Silicon Dioxide-OH nanoparticles was very hydrophilic due to the existence of plentiful hydroxyl sets. Its hydrophilic cooperation with the $\mathrm{H}_{2} \mathrm{O}$-dissolvable segments inside the unique fingermark edges had a significant impact. In the case of OFDBT/Silicon Dioxide$\mathrm{NH}_{2}$ nanoparticles, the quality of images additionally diminished with inundation time, and colossal totals showed up after a more drawn out submersion process. Taking into account that most of the biomolecules having anionic charges[61, 62, 8], the nanoparticles having cationic charges may require a powerful association with them. In this manner, both of the OFDBT/Silicon Dioxide-Epoxy nanoparticles and OFDBT/Silicon Dioxide- $\mathrm{NH}_{2}$ nanoparticles demonstrated less experiencing the $\mathrm{H}_{2} \mathrm{O}$ inundation. Moreover, the OFDBT/Silicon DioxideEpoxy nanoparticles demonstrated a greater bond with the finger impression edges than OFDBT/Silicon Dioxide- $\mathrm{NH}_{2}$ nanoparticles, showing the epoxy bunches additionally added to the high proclivity towards the finger impressions [63].

\section{Conclusions}

Forensic science has many branches and deals with different pieces of evidence which helps in getting the criminal identity, among them fingerprint is one of the significant pieces of evidence when it comes to finding the essential lead regarding the suspect. Fingerprint depicts the individual as fingerprints are unique; no two individual shares the same fingerprints. Apart from the characteristics of the fingerprints are enhanced with proper techniques, one can extract the DNA from it. The nanoparticle development method enhances the latent prints in a better way. By doping the nanoparticles with other compounds such as n- doped carbon dots, Silica Oxide @LaOF: Eu ${ }^{3+}$ core-shell functional nanoparticles lead to enhancement of finger impressions with all the details in it like individual and class characteristics, and location of pores. The exploitation of nanoparticle in latent fingerprinting provided a different direction for forensic investigators. These nanosized particles, when used as a developmental method in the latent fingerprinting, reflect numerous advantages over conventional methods. These particles can easily be employed to reveal fingerprints on various surfaces and has excellent potential for envisioning of finger ridge detailing in a more precise way. This reflects the better discernibility of nanoparticles over commercially available conventional materials used for fingerprinting.

\section{Funding}

This research received no external funding.

\section{Acknowledgments}

This research has no acknowledgment.

\section{Conflicts of Interest}

The authors declare no conflict of interest.

\section{References}

1. Becue, A.; Champod, C.; Margot, P.; Abebe, B.; Chowdappa, H.; Murthy, A.; Zereffa, E.A.; Dessie, Y.; Tang, X.; Huang, L.; Zhang, W.; Zhong, H.; Colleague, D.; Crime, T.; Fund, V.; Tang, X.; Huang, L.; Zhang, 
W.; Zhong, H.; Mohamed, A.A A.; Zhong, K.; Song, K.; Clays, K.; Renn, O.; Roco, M.C.; Yu, C.; Chen, R.; Li, J.J.; Li, J.J.; Drahansky, M.; Paridah, M.; Moradbak, A.; Mohamed, A.A.A.; Owolabi, F.L.H.; Abdulwahab, T.; Asniza, M.; Abdul Khalid, S.H.; Sharma, T.; Dohare, N.; Kumari, M.; Singh, U.K.; Khan, A.B.; Borse, M.S.; Patel, R.; Paez, A.; Howe, A.; Goldschmidt, D.; Corporation, C.; Coates, J.; Reading, F. Use of Gold Nanoparticles as Molecular Intermediates for the Detection of Fingermarks. Anal. Chem. 2015, 44, 2693-2701, https://doi.org/10.1021/ac504693v.

2. Christian, P.; Von Der Kammer, F.; Baalousha, M.; Hofmann, T. Nanoparticles: Structure, Properties, Preparation and Behaviour in Environmental Media. Ecotoxicology 2008, 17, 326-343, https://doi.org/10.1007/s10646-008-0213-1.

3. Rawtani, D.; Tharmavaram, M.; Pandey, G.; Hussain, C.M. Functionalized Nanomaterial for Forensic Sample Analysis. TrAC-Trends Anal. Chem. 2019, 120, https://doi.org/10.1016/j.trac.2019.115661.

4. Abebe, B.; Chowdappa, H.; Murthy, A.; Zereffa, E.A.; Dessie, Y. Latent Fingerprint Enhancement Techniques: A Review. J. Chem. Rev. 2020, 2, 40-56, https://doi.org/10.33945/sami/jcr.2020.1.3.

5. Girod, A.; Ramotowski, R.; Weyermann, C. Composition of Fingermark Residue: A Qualitative and Quantitative Review. Forensic Sci. Int. 2012, 223, 10-24, https://doi.org/10.1016/j.forsciint.2012.05.018.

6. Yan, L.; Yu, Y.; Xia, Z. Microwave-Assisted in Situ Synthesis of Fluorescent Gold Nanoclusters with BSA/Montmorillonite and Application on Latent Fingermark Imaging. Sci. China Chem. 2018, 61, 619-626, https://doi.org/10.1007/s11426-017-9216-7.

7. Li, H.; Wang, T.; Tang, Y.; Wu, J.; Yu, P.; Guo, L.; Chen, J.; Zhang, Y. Combining Multi-Scale Composite Windows with Hierarchical Smoothing Strategy for Fingerprint Orientation Field Computation. Biomed. Eng. Online 2018, 17, 1-21, https://doi.org/10.1186/s12938-018-0559-4.

8. Wang, Y.; Wang, J.; Ma, Q.; Li, Z.; Yuan, Q. Recent Progress in Background-Free Latent Fingerprint Imaging. Nano Res. 2018, 11, 5499-5518, https://doi.org/10.1007/s12274-018-2073-1.

9. Zhao, J.; Zhang, K.; Li, Y.; Ji, J.; Liu, B. High-Resolution and Universal Visualization of Latent Fingerprints Based on Aptamer-Functionalized Core-Shell Nanoparticles with Embedded SERS Reporters. ACS Appl. Mater. Interfaces 2016, 8, 14389-14395, https://doi.org/10.1021/acsami.6b03352.

10. Frens, G. Controlled Nucleation for the Regulation of the Particle Size in Monodisperse Gold Suspensions. Nat. Phys. Sci. 1973, https://doi.org/10.1038/physci241020a0.

11. Tang, X.; Huang, L.; Zhang, W.; Zhong, H. Chemical Imaging of Latent Fingerprints by Mass Spectrometry Based on Laser Activated Electron Tunneling. Anal. Chem. 2015, 87, 2693-2701, https://doi.org/10.1021/ac504693v.

12. Jones, B.J.; Reynolds, A.J.; Richardson, M.; Sears, V.G. Nano-Scale Composition of Commercial White Powders for Development of Latent Fingerprints on Adhesives. Sci. Justice 2010, 50, 150-155, https://doi.org/10.1016/j.scijus.2009.08.001.

13. Moret, S.; Bécue, A.; Champod, C. Functionalised Silicon Oxide Nanoparticles for Fingermark Detection. Forensic Sci. Int. 2016, 259, 10-18, https://doi.org/10.1016/j.forsciint.2015.11.015.

14. Sametband, M.; Shweky, I.; Banin, U.; Mandler, D.; Almog, J. Application of Nanoparticles for the Enhancement of Latent Fingerprints. Chem. Commun. 2007, 11, 1142-1144, https://doi.org/10.1039/b618966k.

15. Choi, M.J.; McDonagh, A.M.; Maynard, P.; Roux, C. Metal-Containing Nanoparticles and Nano-Structured Particles in Fingermark Detection. Forensic Science International. 2008, https://doi.org/10.1016/j.forsciint.2008.04.027.

16. Theaker, B.J.; Hudson, K.E.; Rowell, F.J. Doped Hydrophobic Silica Nano- and Micro-Particles as Novel Agents for Developing Latent Fingerprints. Forensic Sci. Int. 2008, 174, 26-34, https://doi.org/10.1016/j.forsciint.2007.02.030.

17. Chen, B.; Wang, F. Emerging Frontiers of Upconversion Nanoparticles. Trends in Chemistry 2020, https://doi.org/10.1016/j.trechm.2020.01.008.

18. Vetrone, F.; Capobianco, J. A. Lanthanide-Doped Fluoride Nanoparticles: Luminescence, Upconversion, and Biological Applications. Int. J. Nanotechnol. 2008, 5, 1306-1339, https://doi.org/10.1504/IJNT.2008.019840.

19. Chatterjee, D. K.; Gnanasammandhan, M. K.; Zhang, Y. Small Upconverting Fluorescent Nanoparticles for Biomedical Applications. Small 2010, 6, 2781-2795, https://doi.org/10.1002/smll.201000418.

20. Mader, H.S.; Kele, P.; Saleh, S.M.; Wolfbeis, O.S. Upconverting Luminescent Nanoparticles for Use in Bioconjugation and Bioimaging. Current Opinion in Chemical Biology. 2010, 14, 582-596, https://doi.org/10.1016/j.cbpa.2010.08.014.

21. Haase, M.; Schäfer, H. Upconverting Nanoparticles. Angew. Chemie - Int. Ed. 2011, 50, 5808-5829, https://doi.org/10.1002/anie.201005159.

22. Wang, Y.Y.F.; Wang, J.; Ma, Q.; Li, Z.; Yuan, Q.; Shen, J.; Sun, L.D.; Yan, C. H.; Sametband, M.; Shweky, I.; Banin, U.; Mandler, D.; Almog, J.; Rahman, P.; Green, M.; Jin, Y.J.; Luo, Y.J.; Li, G.P.; Li, J.; Wang, Y. Y.F.; Yang, R.Q.; Lu, W.T.; Haase, M.; Schäfer, H.; Girod, A.; Ramotowski, R.; Weyermann, C.; Gao, F.; Lv, C.; Han, J.; Li, X.X.; Wang, Q.; Zhang, J.; Chen, C. M. C.; Li, Q.; Sun, X.; Zheng, J.; Bao, L.; Li, X. X.; Liu, Z.; Gao, D.; Li, F.; Song, J.; Xu, X.; Zhang, Q.; Niu, L.; Dilag, J.; Kobus, H.; Ellis, A.V.; Comi, T.J.; Ryu, S.W.; Perry, R.H.; Chatterjee, D.K.; Gnanasammandhan, M.K.; Zhang, Y.; Cadd, S.; Islam, M.; 
Manson, P.; Bleay, S.; Vetrone, F.; Capobianco, J.A.; Fouad, O.A.; Ismail, A.A.; Zaki, Z.I.; Mohamed, R.M.; Becue, A.; Champod, C.; Margot, P.; Mobaraki, A.; Hosseinzadeh, Z.; Yavari, I.; Yu, I.H.; Jou, S.; Chen, C.M.C.; Wang, K.C.; Pang, L.J.; Liao, J.S. Lanthanide-Doped Fluoride Nanoparticles: Luminescence, Upconversion, and Biological Applications. Forensic Sci. Int. 2011, 22, 5808-5829, https://doi.org/10.1021/acs.analchem.5b03010.

23. Becue, A.; Moret, S.; Champod, C.; Margot, P. Use of Quantum Dots in Aqueous Solution to Detect Blood Fingermarks on Non-Porous Surfaces. Forensic Sci. Int. 2009, 191, 36-41, https://doi.org/10.1016/j.forsciint.2009.06.005.

24. Wang, Y.F.; Yang, R.Q.; Wang, Y.J.; Shi, Z.X.; Liu, J.J. Application of CdSe Nanoparticle Suspension for Developing Latent Fingermarks on the Sticky Side of Adhesives. Forensic Sci. Int. 2009, 185, 96-99, https://doi.org/10.1016/j.forsciint.2008.12.021.

25. Gao, F.; Han, J.; Zhang, J.; Li, Q.; Sun, X.; Zheng, J.; Bao, L.; Li, X.; Liu, Z. The Synthesis of Newly Modified CdTe Quantum Dots and Their Application for Improvement of Latent Fingerprint Detection. Nanotechnology 2011, 22.

26. Shen, J.; Sun, L.D.; Yan, C.H. Luminescent Rare Earth Nanomaterials for Bioprobe Applications. Dalt. Trans. 2008, 9226, 5687-5697, https://doi.org/10.1039/b805306e.

27. Wang, M.; Zhu, Y.; Mao, C. Synthesis of NIR-Responsive NaYF4:Yb,Er Upconversion Fluorescent Nanoparticles Using an Optimized Solvothermal Method and Their Applications in Enhanced Development of Latent Fingerprints on Various Smooth Substrates. Langmuir 2015, 31, 7084-7090, https://doi.org/10.1021/acs.langmuir.5b01151.

28. Mobaraki, A.; Hosseinzadeh, Z.; Yavari, I. Lipophilic Magnetic Nanocomposite of Fe3O4@SiO2@Me for Efficient Visualization of Latent Fingerprints on Various Surfaces. J. Iran. Chem. Soc. 2019, 16, 1601-1610, https://doi.org/10.1007/s13738-019-01636-z.

29. Nandhini, G.; Suriyaprabha, R.; Maria Sheela Pauline, W.; Rajendran, V.; Aicher, W. K.; Awitor, O. K. Influence of Solvents on the Changes in Structure, Purity, and in Vitro Characteristics of Green-Synthesized ZnO Nanoparticles from Costus Igneus. Appl. Nanosci. 2018, 8, 1353-1360, https://doi.org/10.1007/s13204018-0810-0.

30. Choi, M.J.; McDonagh, A.M.; Maynard, P.J.; Wuhrer, R.; Lennard, C.; Roux, C. Preparation and Evaluation of Metal Nanopowders for the Detection of Fingermarks on Nonporous Surfaces. J. Forensic Identif. 2006.

31. Madhavan, A.A.; Kumar Sharma, B. Latent Fingerprint Development with Biosynthesized Nano Rust. 2019 Adv. Sci. Eng. Technol. Int. Conf. ASET 2019, 2019, 1-4, https://doi.org/10.1109/ICASET.2019.8714290.

32. Prasad, V.; Lukose, S.; Agarwal, P.; Prasad, L. Role of Nanomaterials for Forensic Investigation and Latent Fingerprinting-A Review. J. Forensic Sci. 2020, 65, 26-36, https://doi.org/10.1111/1556-4029.14172.

33. Fernandes, D.; Krysmann, M. J.; Kelarakis, A. Carbon Dot Based Nanopowders and Their Application for Fingerprint Recovery. Chem. Commun. 2015, 51, 4902-4905, https://doi.org/10.1039/c5cc00468c.

34. Mukherjee, A.; Adak, M. K.; Dhak, P.; Dhak, D. A Simple Chemical Method for the Synthesis of Cu2+ Engrafted MgAl2O4 Nanoparticles: Efficient Fluoride Adsorbents, Photocatalyst and Latent Fingerprint Detection. J. Environ. Sci. (China) 2020, 88, 301-315, https://doi.org/10.1016/j.jes.2019.09.004.

35. Zhong, K.; Song, K.; Clays, K. Hollow Spheres: Crucial Building Blocks for Novel Nanostructures and Nanophotonics. Nanophotonics 2018, 7, 693-713, https://doi.org/10.1515/nanoph-2017-0109.

36. Bharat, L.K.; Raju, G.S.R.; Yu, J.S. Red and Green Colors Emitting Spherical-Shaped Calcium Molybdate Nanophosphors for Enhanced Latent Fingerprint Detection. Sci. Rep. 2017, 7, 1-14, https://doi.org/10.1038/s41598-017-11692-1.

37. Suresh, C.; Nagabhushana, H.; Basavaraj, R.B.; Darshan, G.P.; Kavyashree, D.; Daruka Prasad, B.; Sharma, S.C.; Vanithamani, R. SiO2@LaOF:Eu3+ Core-Shell Functional Nanomaterials for Sensitive Visualization of Latent Fingerprints and WLED Applications. J. Colloid Interface Sci. 2018, 518, 200-215, https://doi.org/10.1016/j.jcis.2018.01.093.

38. Moret, S.; Bécue, A.; Champod, C. Nanoparticles for Fingermark Detection: An Insight into the Reaction Mechanism. Nanotechnology 2014, 25.

39. Li, J.; Zhu, X.; Xue, M.; Feng, W.; Ma, R.; Li, F. Nd3+-Sensitized Upconversion Nanostructure as a DualChannel Emitting Optical Probe for Near Infrared-to-Near Infrared Fingerprint Imaging. Inorg. Chem. 2016, 55, 10278-10283, https://doi.org/10.1021/acs.inorgchem.6b01536.

40. Lin, S.S.; Yemelyanov, K.M.; Pugh, Jr.E.N.; Engheta, N. Polarization-Based and Specular-Reflection-Based Noncontact Latent Fingerprint Imaging and Lifting. J. Opt. Soc. Am. A 2006, 23, https://doi.org/10.1364/josaa.23.002137.

41. Milenkovic, I.; Algarra, M.; Alcoholado, C.; Cifuentes, M.; Lázaro-Martínez, J.M.; Rodríguez-Castellón, E.; Mutavdžić, D.; Radotić, K.; Bandosz, T.J. Fingerprint Imaging Using N-Doped Carbon Dots. Carbon N. Y. 2019, 144, 791-797, https://doi.org/10.1016/j.carbon.2018.12.102.

42. Qin, G.; Zhang, M.; Zhang, Y.; Zhu, Y.; Liu, S.; Wu, W.; Zhang, X. Visualizing Latent Fingerprints by Electrodeposition of Metal Nanoparticles. J. Electroanal. Chem. 2013, 693, 122-126, https://doi.org/10.1016/j.jelechem.2013.01.016.

43. Welch, C.M.; Compton, R.G. The Use of Nanoparticles in Electroanalysis: A Review. Anal. Bioanal. Chem. 2006, 384, 601-619, https://doi.org/10.1007/s00216-005-0230-3. 
44. Wang, J.; Wang, L.; Di, J.; Tu, Y. Electrodeposition of Gold Nanoparticles on Indium/Tin Oxide Electrode for Fabrication of a Disposable Hydrogen Peroxide Biosensor. Talanta 2009, 77, 1454-1459, https://doi.org/10.1016/j.talanta.2008.09.034.

45. Xiao, Y.; Patolsky, F.; Katz, E.; Hainfeld, J.F.; Willner, I. Plugging into Enzymes: Nanowiring of Redox Enzymes by a Gold Nanoparticle. Science (80-). 2003, 299 1877-1881, https://doi.org/10.1126/science.1080664.

46. Arshad, A.; Farrukh, M.A.; Ali, S.; Khaleeq-ur-Rahman, M.; Tahir, M.A. Development of Latent Fingermarks on Various Surfaces Using ZnO-SiO2 Nanopowder. J. Forensic Sci. 2015, 60, 1182-1187, https://doi.org/10.1111/1556-4029.12890.

47. Luthra, D.; Kumar, S. The Development of Latent Fingerprints by Zinc Oxide and Tin Oxide Nanoparticles Prepared by Precipitation Technique. AIP Conf. Proc. 2018, 1953, https://doi.org/10.1063/1.5032584.

48. Jones, N.; Stoilovic, M.; Lennard, C.; Roux, C. Vacuum Metal Deposition: Developing Latent Fingerprints on Polyethylene Substrates after the Deposition of Excess Gold. Forensic Sci. Int. 2001, 123, 5-12, https://doi.org/10.1016/S0379-0738(01)00507-2.

49. Kim, S.S.; Lee, B.T. Effects of Oxygen Pressure on the Growth of Pulsed Laser Deposited ZnO Films on Si(0 0 1). Thin Solid Films 2004, 446, 307-312, https://doi.org/10.1016/j.tsf.2003.09.057.

50. Fouad, O.A.; Ismail, A.A.; Zaki, Z.I.; Mohamed, R.M. Zinc Oxide Thin Films Prepared by Thermal Evaporation Deposition and Its Photocatalytic Activity. Appl. Catal. B Environ. 2006, 62, 144-149, https://doi.org/10.1016/j.apcatb.2005.07.006.

51. Bouhssira, N.; Abed, S.; Tomasella, E.; Cellier, J.; Mosbah, A.; Aida, M.S.; Jacquet, M. Influence of Annealing Temperature on the Properties of ZnO Thin Films Deposited by Thermal Evaporation. Appl. Surf. Sci. 2006, 252, 5594-5597, https://doi.org/10.1016/j.apsusc.2005.12.134.

52. Jones, N.; Mansour, D.; Stoilovic, M.; Lennard, C.; Roux, C. The Influence of Polymer Type, Print Donor and Age on the Quality of Fingerprints Developed on Plastic Substrates Using Vacuum Metal Deposition. Forensic Sci. Int. 2001, 124, 167-177, https://doi.org/10.1016/S0379-0738(01)00593-X.

53. Philipson, D.; Bleay, S. Erratum: Alternative Metal Processes for Vacuum Metal Deposition. Journal of Forensic Identification 2007, 57, 252-273.

54. Yu, I.H.; Jou, S.; Chen, C.M.; Wang, K.C.; Pang, L.J.; Liao, J.S. Development of Latent Fingerprint by ZnO Deposition. Forensic Sci. Int. 2011, 207, 14-18, https://doi.org/10.1016/j.forsciint.2010.07.035.

55. Mohamed, A.A. Gold Is Going Forensic. Gold Bull. 2011, 44, 71-77, https://doi.org/10.1007/s13404-0110013-x.

56. Gao, D.; Li, F.; Song, J.; Xu, X.; Zhang, Q.; Niu, L. One Step to Detect the Latent Fingermarks with Gold Nanoparticles. Talanta 2009, 80, 479-483, https://doi.org/10.1016/j.talanta.2009.07.007.

57. Murray, R. W. Nanoparticles: An Emerged and Lasting Frontier. Anal. Chem. 2009, 81, https://doi.org/10.1021/ac900238p.

58. Wilson, R. The Use of Gold Nanoparticles in Diagnostics and Detection. Chem. Soc. Rev. 2008, 37, 20282045, https://doi.org/10.1039/b712179m.

59. Ni, W.; Kou, X.; Yang, Z.; Wang, J. Tailoring Longitudinal Surface Plasmon Wavelengths, Scattering and Absorption Cross Sections of Gold Nanorods. ACS Nano 2008, 2, 677-686, https://doi.org/10.1021/nn7003603.

60. Tang, H.W.; Lu, W.; Che, C.M.; Ng, K.M. Gold Nanoparticles and Imaging Mass Spectrometry: Double Imaging of Latent Fingerprints. Anal. Chem. 2010, 82, 1589-1593, https://doi.org/10.1021/ac9026077.

61. Comi, T.J.; Ryu, S.W.; Perry, R.H. Synchronized Desorption Electrospray Ionization Mass Spectrometry Imaging. Anal. Chem. 2016, 88, 1169-1175, https://doi.org/10.1021/acs.analchem.5b03010.

62. Cadd, S.; Islam, M.; Manson, P.; Bleay, S. Fingerprint Composition and Aging: A Literature Review. Sci. Justice 2015, 55, 219-238, https://doi.org/10.1016/j.scijus.2015.02.004.

63. Yang, Y.; Liu, R.; Cui, Q.; Xu, W.; Peng, R.; Wang, J.; Li, L. Red-Emissive Conjugated Oligomer/Silica Hybrid Nanoparticles with High Affinity and Application for Latent Fingerprint Detection. Colloids Surfaces A Physicochem. Eng. Asp. 2019, 565, 118-130, https://doi.org/10.1016/j.colsurfa.2019.01.009. 